\title{
Coup d'œil sur les petites GTPases Rho
}

> Les premiers membres identifiés de la sous-

\section{Martin Primeau, Nathalie Lamarche-Vane}

famille des petites GTPases Rho, soit RhoA, Racl et $\mathrm{Cdc} 42$, ont fait l'objet d'intenses recherches depuis les premières indications du rôle primordial qu'elles jouent dans la réorganisation du cytosquelette d'actine. Depuis, les recherches sur ces protéines ont grandement accru nos connaissances dans les secteurs de la polarisation et de la migration cellulaire, auxquels il faut ajouter celui du transport des vésicules. Vingt gènes codant des GTPases associées à cette famille dans le génome humain ont maintenant été répertoriés. Si nos connaissances sur ces autres GTPases Rho demeurent encore fragmentaires, leur étude nous confirme que chacune exerce une fonction spécifique rendant ainsi leur présence pratiquement indispensable au fonctionnement normal d'un organisme. Dans cet article, nous tentons de dresser un bref état de nos connaissances sur les diverses fonctions qu'occupe au sein de la cellule l'ensemble des GTPases Rho. <

Dans leur effort d'adaptation à leur environnement, les cellules eucaryotes ont dû se spécialiser et surtout se doter d'outils pour exécuter à de faibles coûts énergétiques des processus biologiques complexes. La conservation du module GTPase chez les eucaryotes en constitue un bel exemple. Un sous-groupe de petites protéines $\mathrm{G}$ monomériques rassemblées sous le nom de superfamille Ras compte chez l'humain plus de 150 membres subdivisés en cinq sous-familles principales (Arf, Rab, Ran, Ras et Rho) ayant chacune son propre champ de spécialisation (Tableau I).

Bien qu'elles contribuent de près ou de loin à remplir une gamme de fonctions dans la cellule, on reconnaît aux GTPases Rho un rôle prépondérant dans la polarisation de structures cellulaires, la structuration de voies de signalisation et l'organisation du cytosquelette [1]. Chez I'humain, on trouve 22 membres de cette famille codés par 20 gènes (Figure 1) [2]. Chaque membre comprend un

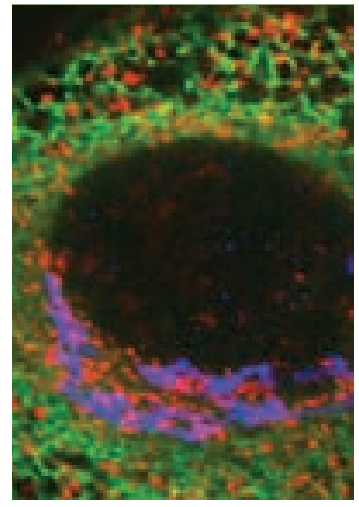

Université McGill, Département d'anatomie et biologie cellulaire, 3640 University, room 1-38, Montréal, Québec, H3A 2B2 Canada. nathalie.lamarche@mcgill.ca

domaine $G$ commun aux GTPases de la superfamille Ras d'environ 160 acides aminés qui agit comme interrupteur et temporisateur du signal selon qu'il lie un nucléotide guanylique GTP ou GDP (Figure 2) [3]. Cependant, les membres de la famille Rho se distinguent par la présence d'une séquence insert de 12 acides aminés localisée entre le cinquième feuillet $\beta$ et la quatrième hélice $\alpha$ du domaine GTPase [4]. Le domaine $G$ est, à l'exception des deux membres de la famille RhoBTB, le seul domaine globulaire trouvé dans ces protéines.

Pour agir efficacement comme intermédiaire de la transmission de l'information, la majorité des GTPases Rho sont amarrées aux membranes par une attache lipidique ajoutée de façon post-traductionnelle à leur extrémité carboxy-terminale selon qu'on y trouve ou non une séquence de quatre acides aminés appelée boîte CAAX. Celle-ci est reconnue par la farnésyl-transférase et/ou une géranylgéranyl-transférase selon la nature des acides aminés composant le segment (Tableau II). Ces enzymes catalysent l'addition d'un groupement isoprényl (farnésyl «F» ou géranylgéranyl « $G G$ » respectivement) sur la chaîne latérale de la cystéine de la boîte CAAX. Les acides aminés en aval de la cystéine sont ensuite clivés et son groupement carboxyl est méthylé. Outre la présence de la boîte CAAX, une région riche en résidus basiques (arginines et lysines), située en amont du signal de prénylation, constitue aussi une caractéristique des GTPases. Si la fonction de cette région est de stabiliser la liaison des GTPases aux membranes, elle peut aussi contribuer à lier certaines protéines ou encore à agir comme signal de localisation nucléaire (voir résumé dans [5]). 


\begin{tabular}{ccc}
\hline $\begin{array}{c}\text { Sous- } \\
\text { famille }\end{array}$ & $\begin{array}{c}\text { Nombre } \\
\text { de gènes par } \\
\text { sous-famille }\end{array}$ & \multicolumn{1}{c}{ Rôle fonctionnel } \\
\hline Arf & 27 & Formation et trafic de vésicules \\
\hline Rab & 61 & Trafic de vésicules \\
\hline Ran & 1 & Transport nucléaire \\
\hline Ras & 36 & $\begin{array}{l}\text { Contrôle de la prolifération } \\
\text { et différenciation cellulaire }\end{array}$ \\
\hline Rho & 20 & $\begin{array}{l}\text { Contrôle de la prolifération } \\
\text { et morphologie cellulaire }\end{array}$ \\
\hline
\end{tabular}

Tableau I. Membres de la superfamille des petites GTPases Ras.

La majorité des publications ayant eu pour sujet les GTPases Rho ont porté par le passé sur l'étude de $C d c 42, \operatorname{Racl}$ et RhoA. Les dernières années ont toutefois permis de mettre en lumière le rôle distinct des 19 autres protéines de cette famille. Le tour d'horizon que propose cet article a pour objectif de tenter d'exposer globalement les diverses fonctions cellulaires attribuées jusqu'à maintenant à la plupart de ces petites GTPases.

\section{RhoA, RhoB et RhoC}

Le groupe des trois membres de la famille Rho (RhoA, RhoB et RhoC) s'impose comme un exemple frappant de divergence évolutive chez les mammifères. RhoA et RhoC partagent entre elles une identité structurelle de $92 \%$ et de $82 \%$ avec RhoB. Chacune stimule la contractilité des filaments d'actine par la myosine Iorsqu'on l'exprime sous sa forme constitutivement active dans des fibroblastes, ce qui suggère le recours aux mêmes effecteurs. Ces GTPases se distinguent entre elles toutefois par la nature des acides aminés de leur extrémité carboxy-terminale (Tableau II). En conséquence, tandis que RhoA et RhoC sont géranylgéranylées, RhoB voit sa dernière cystéine alternativement modifiée par l'ajout d'un «F » ou d'un «GG » [6], tout en étant palmitoylée sur deux cystéines en amont du site de prénylation.

Si l'homologie de ces protéines est élevée, leur rôle dans le cancer est variable. L'expression de RhoA ou de RhoC s'intensifie dans plusieurs types de tumeurs [7]. RhoA est aussi connue pour sa contribution à la progression du cycle cellulaire. Sous sa forme liant le GTP, elle bloque l'activation de $\mathrm{p} 21^{\text {Wafl/Cipl }}$ par Ras, favorisant le passage du cycle de la phase Gl à la phase $S$ [8]. Inversement, le gène RhoB est perçu comme un suppresseur de tumeurs : son expression tend à décrồtre avec l'évolution de plusieurs types de tumeurs [9]. Si RhoA et RhoC sont exprimées de façon ubiquitaire, comme les formes protéique et ribonucléique de RhoB, elles n'ont que de faibles demi-vies et leur expression n'est augmentée qu'en réponse à un stress cellulaire ou à une stimulation par des facteurs de croissance, particulièrement au cours des phases $\mathrm{Gl}$ et $\mathrm{S}$ du cycle cellulaire [10].

\section{Rac1, Rac2, Rac3 et RhoG}

Racl, Rac2 et Rac3 ont une similarité supérieure à $95 \%$ tandis que RhoG ne montre que $80 \%$ de similitude. Comme pour les membres de la sous-famille de RhoA, ces protéines tirent leur principale distinction de leur région basique carboxy-terminale (Tableau II). Elles renferment des acides aminés leur permettant de lier de façon spécifique certaines protéines. Par exemple, bien que Racl et Rac2 lient toutes deux la kinase Pakl, Racl est plus apte que Rac2 à lier et à activer Pak1 [11]. La transmutation des régions basiques respectives des deux GTPases annule la propriété des GTPases à lier Pakl. Dans le même ordre d'idée, la capacité de Rac3 à lier la

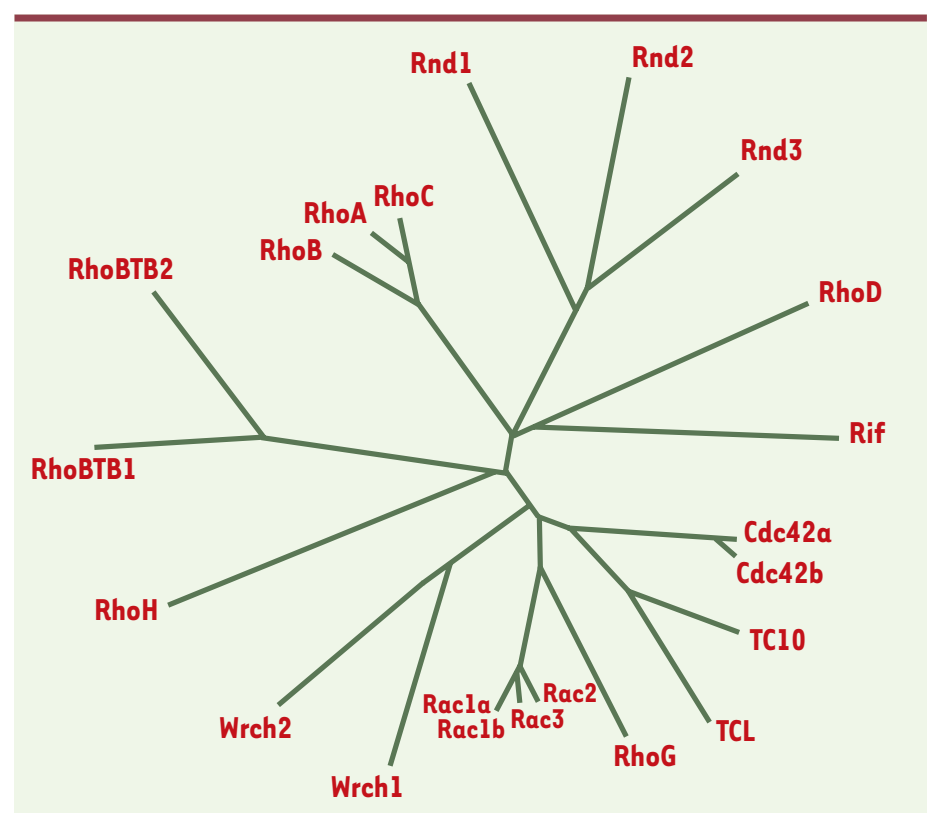

Figure 1. Arbre phylogénique des GTPases de la famille Rho chez Homo sapiens. Vingt gènes codent des GTPases appartenant à la famille Rho. Dans ce lot, Racl, RhoA et Cdc42 ont fait l'objet d'études pionnières. Chacune de ces protéines régule à sa façon la dynamique du cytosquelette. L'activation de Racl entraîne la formation de longs feuillets nommés lamellipodes et aurait un rôle central dans la migration des cellules; l'activation de RhoA conduit au fagotage des filaments d'actine en fibres de stress; Cdc42, sous sa forme active, est associée à la formation de projections cellulaires constituées de filaments d'actine appelés filopodes. Cette propriété se traduit biologiquement par un rôle dans la polarisation de structures cellulaires comme la croissance des axones ou la migration cellulaire (les GTPases de la famille Rho ont été alignées par Clustal W et l'arbre réalisé avec Treeview). 
protéine CIB (Calmodulin- and integrin-binding protein) par l'intermédiaire de sa région carboxy-terminale n'est pas une fonction dont peut s'affranchir Racl [12].

Bien qu'elles aient une grande similarité, les protéines de ce groupe ont avant tout des patrons d'expression distincts. Si Racl et Rac3 sont exprimées de façon ubiquitaire, Rac2, elle, est restreinte aux tissus hématopoïétiques. L'absence de Rac2 chez les souris provoque d'ailleurs des troubles du développement du système hématopoïétique [13]. Racl existe aussi sous une forme alternative, Raclb, découverte à l'origine dans des cellules cancéreuses [7]. Cette isoforme provient d'un épissage alternatif qui produit une insertion de 19 acides aminés tout juste après la région «switch 2 », rendant Raclb constitutivement active. Rac3 pourrait également contribuer à la formation de tumeurs. Par exemple, son activation induit la prolifération de cellules cancéreuses du sein [7]. On ne connaît pas de fonction du même genre pour RhoG. Toutefois, sous sa forme liant le GTP, elle active le complexe ELMO-Dock180, entraînant l'activation de Racl [14]. On reconnaît aussi une contribution de RhoG aux mécanismes de phagocytose par les macrophages de cellules apoptotiques [15].

\section{Cdc42, TC10, TCL}

TC10 et TCL sont les plus proches parents de Cdc42. Bien que toutes trois partagent un bon nombre d'effecteurs communs et implicitement partagent la capacité d'induire la formation de filopodes dans les fibroblas-

\begin{tabular}{|c|c|c|c|}
\hline GTPases & Séquence carboxy-terminale & Prénylation & Palmitoylation \\
\hline $\operatorname{Cdc} 42 a$ & -PPEPKKSRRCVLL ${ }^{1}$ & $\mathrm{GG}^{2}$ & \\
\hline Cdc42b & -PPETQPKRKCCIF & GG & \\
\hline $\mathrm{TClO}$ & -PKKHTVKKRIGSRCINCCLIT & $F ?^{3}$ & oui \\
\hline $\mathrm{TCL}$ & -PKKKKKRCSEGHSCCSIII & $\mathrm{F}$ ? & oui? \\
\hline Wrchl & -YSDTQ९QPKKSKSRTPDKMKNLSKSWWKKYCCFV & Non & oui \\
\hline Wrch2 & -HKARLEKKLNAKGVRTLSRCRWKKFFCFV & Non & oui \\
\hline $\operatorname{Racl}^{4}$ & -PPPVKKRKRKCLLLL & GG & \\
\hline Rac2 & -PQPTRQQKRACSLL & GG & \\
\hline Rac3 & -PPPVKKPGKKCTVF & GG & \\
\hline RhoG & -PTPIKRGRSCILL & GG & \\
\hline RhoA & -ARRGKKKSGCLLL & GG & \\
\hline RhoB & -KRYGSPNGCINCCKVL & $G G / F$ & oui \\
\hline RhoC & -VRKNKRRRGCPIL & GG & \\
\hline Rndl & -KPSPLPQKSPVRSLSKRLLHLPSRSELISSTFKKEKAKSCSIM & $\mathrm{F}$ & \\
\hline Rnd2 & -RGHRQLRRTDSRRGMQRSAQLSGRPDRGNEGEIHKDRAKSCNLM & $\mathrm{F}$ & \\
\hline Rnd3 & -KTNKNVKRNKSQRATKRISHMPSRPELSAVATDLRKDKAKSCTVM & $\mathrm{F}$ & \\
\hline RhoD & -SRGRNFWRRITQGFCVVT & $\mathrm{F} ?$ & \\
\hline Rif & -ALKKA@RQKKRRLCLLL & GG? & \\
\hline RhoBTBl & Domaine GTPase en amino-terminal de la protéine & Non & \\
\hline RhoBTB2 & Domaine GTPase en amino-terminal de la protéine & Non & \\
\hline RhoH/TTF & -QARRRNRRRLFSINECKKIF & GG & \\
\hline
\end{tabular}

Tableau II. Caractéristiques majeures de la région carboxy-terminale des Rho GTPases.

${ }^{1}$ Résidus basiques (gras) ; cystéines prénylées (souligné); cystéines palmitoylées (italique).

${ }^{2} \mathrm{GG}$ : géranylgéranyl ; $\mathrm{F}$ : farnésyl. ${ }^{3}$ Le point d'interrogation souligne l'absence d'une démonstration de la modification prédite.

${ }^{4}$ Les deux isoformes de Racl sont identiques au niveau de leur région carboxy-terminale. 
tes, ces GTPases comportent des ancres lipidiques distinctes. Si Cdc42 est strictement géranylgéranylée en son extrémité carboxy-terminale, TC10 et TCL comprennent une boîte CAAX potentiellement reconnue par la farnésyl-transférase. De plus, deux cystéines additionnelles situées dans la portion carboxy-terminale de TC10 et TCL agissent comme sites de palmitoylation. Ces groupements lipidiques préviennent le retrait de TC10 des membranes par le truchement de Rho-GDI-1 [16].

Comme pour $\mathrm{Cdc} 42$, on prête à ces protéines un rôle dans la formation et la régénération d'extensions neuronales. Des expériences menées chez des rats ayant subi un endommagement des nerfs périphériques [17] ou de la colonne vertébrale [18] montrent une augmentation de l'expression de l'ARN messager de TC10 dans les neurones en régénération. De plus, TC10 intervient dans le trafic du canal de glucose GLUT4. À la suite de la stimulation d'adipocytes par de l'insuline, TC10 est activée et favorise l'expression du canal de glucose GLUT4 à la membrane par stimulation de son exocytose [19].

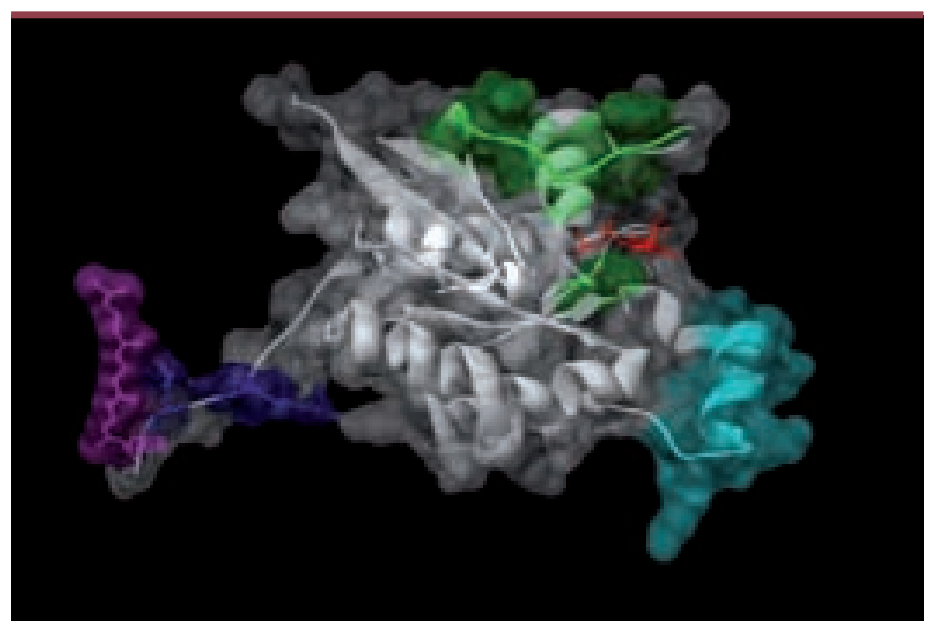

Figure 2. Caractéristiques structurales des GTPases de la famille Rho. Chaque membre de la famille comporte une séquence « insert » de 12 acides aminés située entre le $5^{\mathrm{e}}$ feuillet $\beta$ et la $4^{\mathrm{e}}$ hélice $\alpha$ du domaine GTPase (cyan). Le positionnement des régions Switch I et Switch II (vert) est dépendant de la nature du guanosine (rouge) lié par la GTPase. La majorité des GTPases Rho sont des commutateurs moléculaires existant sous deux états, liant de façon spécifique leurs partenaires d'interaction selon qu'ils soient associés au guanosine-diphosphate (GDP) ou au guanosine-triphosphate (GTP). Le rythme d'échange du GDP pour un GTP est accéléré par des facteurs d'échange de guanines (GEF) qui rendent les GTPases aptes à lier plus efficacement leurs effecteurs. En contrepartie, les GTPases pallient leur capacité enzymatique réduite à hydrolyser le GTP en GDP par l'assistance entre autre d'une arginine spécifique trouvée dans le site de liaison de protéines activatrices de GTPases (GAP). À l'exception de RhoBTB l et 2, le domaine G comporte une modification lipidique à son extrémité carboxylée (magenta) précédée de résidus à chaîne latérale basique (bleu). La modification lipidique ne garantit pas la présence des GTPases dans la membrane. Les GTPases Rho se trouvent généralement séquestrées dans le cytoplasme par des inhibiteurs de la dissociation du GDP (GDI) qui dissimulent notamment la chaîne lipidique des GTPases. Dans le cas présent, Cdc42 lie le GDP et adopte une conformation lui permettant de lier RhoGDI (Protein Data Bank 1DOA) (image réalisée avec UCSF Chimera).
TCL diffère de TC10 principalement par les séquences adjacentes à son domaine GTPase. TCL intervient dans le trafic de récepteurs membranaires; elle est requise pour le transport des protéines contenues dans les vésicules endocytiques vers les endosomes précoces avant d'être recyclées à la membrane [20]. Des protéines chimériques de TC10 ou de Cdc42 munies de la séquence carboxy-terminale de TCL sont également en mesure d'accomplir cette fonction, suggérant ainsi que le module GTPase de ces homologues soit interchangeable et que cette fonction de TCL ne soit déterminée que par la singularité de sa séquence carboxy-terminale [20].

\section{Wrch-1 et Wrch-2}

Wrch-1 et Wrch-2 sont des homologues plus distants de Cdc42. Wrch-1 a été découvert au cours de travaux de repérage des gènes dont l'expression était augmentée en réponse à l'activation de Frizzled par le facteur de croissance Wnt-1. L'activation de cette voie importante pour le développement embryonnaire favorise également la formation de tumeurs. D'ailleurs, la surexpression de Wrch-1 ou d'une forme mutante active de Wrch1 dans des cellules épithéliales mammaires reproduit la transformation de ces cellules par Wnt-1 [21]. Wrch2/Chp a aussi la propriété d'induire la transformation de fibroblastes lorsqu'elle est surexprimée [22]. Wrch-1 et Wrch-2 se distinguent principalement de Cdc42 par la présence sur l'extrémité amino-terminale d'une séquence riche en prolines. Celle-ci permet à Wrch-1 de lier la protéine d'échafaudage Grb2 et d'être activée par elle [23]. Bien que Wrch-1 comporte aussi une boîte CAAX, cette protéine n'est pas prénylée. Comme Wrch2/Chp, elle est uniquement palmitoylée [22], ce qui confère à ces protéines une distribution intracellulaire distincte de celle des autres GTPases Rho.

\section{Rnd1, Rnd2 et Rnd3}

Les Rnd sont de proches parents des protéines de la famille Rho et ont fait récemment l'objet d'une synthèse très complète [24]. Leur incapacité à lier le GDP et à hydrolyser le GTP $[25,26]$ font en sorte qu'elles lient de façon constitutive le GTP et restent insensibles à l'action de GEF ou de GAP. Elles se distinguent également des autres membres de la famille par la présence d'un groupement «F» et non d'un «GG» à leur extrémité carboxy-terminale [25]. Cette absence d'un groupement «GG» leur permet d'échapper à la séquestration cytoplasmique des RhoGDI et explique qu'on retrouve ces protéines principalement localisées aux membranes $[25,26]$. 
Les Rnd agissent comme antagonistes des protéines de la famille Rho. La surexpression de Rndl et de Rnd3 dans les fibroblastes amène ces cellules à perdre leurs points d'ancrage et à s'arrondir [26]. Cette observation correspond précisément au phénotype inverse de RhoA. D'ailleurs, Rndl et Rnd3 lient et activent p190RhoGAP, pour inactiver RhoA [27]. Rnd3 perturbe également la signalisation de RhoA en liant et inhibant un de ses effecteurs, la kinase ROCK [24]. ROCK phosphoryle à son tour Rnd3 et contribue ainsi à augmenter sa demivie protéique.

L'expression de Rnd3 est ubiquitaire; tel n'est pas le cas de Rnd2 qui réside uniquement dans les testicules ni de Rndl principalement localisée, chez l'adulte, dans le cerveau et le foie [26]. Rndl participe d'ailleurs à la rétraction du cône de croissance d'un axone lorsque celui-ci exprime le récepteur Plexine-Bl, une plexine induisant la chimiorépulsion lorsqu'elle s'enchaîne à son substrat, la sémaphorine Sema-4D [28].

\section{RhoD et Rif}

Bien qu'elle présente peu d'homologie avec Cdc42 (40\% de similarité), la protéine RhoD est tout aussi apte à former des filopodes lorsqu'elle est surexprimée sous sa forme liant constitutivement le GTP [29]. Le même mutant affecte également le transport des endosomes précoces et leur fusion en endosomes tardifs. Toutefois, à la différence de $\mathrm{Cdc} 42$, RhoD n'agit pas sur les effecteurs WASP (Wiskott-Aldrich syndrome protein) et $\mathrm{N}$-WASP, mais lie plutôt hDia2C, une isoforme de la formine hDia2 [30]. Comme hDia2, hDia2C lie, une fois activée par la liaison d'une GTPase Rho, l'extrémité positive d'un filament d'actine et permet son élongation. En recrutant hDia2C aux endosomes, RhoD permet l'alignement des endosomes sur les filaments d'actine et exerce donc un rôle dans le trafic intracellulaire des endosomes.

Le plus proche homologue de RhoD est Rif. Comme RhoD et $\mathrm{Cdc42}$, Rif induit de longs filopodes lorsqu'on l'exprime sous une forme liant constitutivement le GTP [31], et ce indépendamment de la présence de Cdc42 [29]. Rif active aussi mDia2, l'orthologue de la formine hDia2. On ignore toutefois quel rôle précis joue Rif dans la cellule.

Rif et RhoD ne comportent pas d'éléments distinctifs dans la région carboxy-terminale et sont isoprénylées comme la plupart des autres GTPases Rho. Toutes deux cependant, comparativement à $\mathrm{Cdc} 42$, comprennent une extension d'environ 15 acides aminés dans la région amino-terminale ; celle-ci pourrait jouer un rôle dans la liaison d'activateurs et/ou d'inhibiteurs qui leur sont spécifiques.

\section{Famille de RhoBTB}

Les gènes de la famille RhoBTB ont été décelés lors d'une recherche d'homologues de Rho chez Dictyostelium discoideum [32]. Les deux membres trouvés chez l'humain sont dotés d'une organisation modulaire particulière: celle-ci débute par un domaine GTPase suivi de deux domaines BTB (Broad-Complex, Tramtrack et Bric-à-Brac) assez courants dans certains facteurs de transcription. Les membres de la famille RhoBTB ne comportent pas de signal de prénylation à l'extrémité carboxy-terminale, ce qui n'empêche pas RhoBTBl et RhoBTB2/ DBC2 de cohabiter avec des structures vésiculaires [29]. La fonction précise de ces protéines demeure inconnue ; une étude récente suggère cependant que la variation du niveau d'expression de RhoBTB2/DBC2 peut avoir un effet sur l'expression de gènes qui participent à la progression du cycle cellulaire, l'apoptose, ainsi que le trafic membranaire et l'organisation du cytosquelette [33]. À cet égard, RhoBTB serait un gène suppresseur de tumeurs: il se trouve muté ou non exprimé dans certains cas de cancer du sein [2]. L'expression de RhoBTB à certains stades du développement embryonnaire laisse supposer aussi que ce gène exercerait une action au cours du développement du système nerveux [34].

\section{RhoH/TTF}

RhoH/TTF (translocation three four) a été originalement découverte dans un transcrit chimérique provenant de la recombinaison entre les chromosomes 3 et 4 décelée chez un patient atteint d'un lymphome non-hodgkinien [7]. Son expression est restreinte aux cellules hématopoïétiques; comme pour les Rnd, elle lie de façon constitutive le GTP pour agir comme antagoniste des fonctions des autres GTPases Rho. Dans le lymphocyte, son expression permet le maintien de la cellule dans un état inactif en favorisant l'état d'adhésion des intégrines LFA1 aux molécules d'adhésion inter-cellulaires (ICAM) [35].

\section{Conclusion}

Les GTPases, en général, et les GTPases Rho, en particulier, illustrent de façon probante la notion de plasticité moléculaire. La somme d'un faible nombre de variations dans la séquence primaire de ces protéines suffit à modifier l'usage que fait la cellule de celles-ci. La grande variabilité des régions carboxy-terminales des GTPases Rho contribue en partie à cette distinction. Le rôle précis de la plupart des membres de cette famille reste cependant à déterminer. C'est pourquoi, il s'avèrera crucial d'identifier au cours des prochaines années les régulateurs spécifiques de chacune de ces GTPases Rho afin de mieux comprendre l'importance de leur activation et le champ de leur fonction, compte tenu du déroulement des processus biologiques dans lesquels elles sont engagées. $\diamond$

\section{SUMMARY}

A brief overview of the small Rho GTPases

RhoA, Racl, and Cdc42, the founding members of the Rho subfamily of small GTPases, have been the focus of many research studies since the 
first discovery of their primary roles in the reorganisation of the actin cytoskeleton. Since then, it is clear that they are involved in a great deal of cellular functions, including cell migration and adhesion, cell growth control, and membrane trafficking. The complete sequencing of the human genome has now highlighted a total of 20 genes encoding Rho-like proteins. Little is known about their distinct cellular functions, however, numerous studies are now beginning to unravel that each of the Rho GTPase must play a specific role in the cell in a timely and spatially regulated fashion. Here, we are presenting a brief overview of the distinct functional roles and similarities known to date for each of the Rho members. $\diamond$

\section{REMERCIEMENTS}

Étant donné l'abondance de la documentation sur les petites GTPases Rho, nous nous excusons si des références pertinentes à ce texte ont été omises à cause du manque d'espace. Martin Primeau et Nathalie Lamarche-Vane sont titulaires, respectivement, d'une bourse d'études supérieures du Canada et d'une bourse de nouveau chercheur des Instituts de recherche en santé du Canada.

\section{RéFÉRENCES}

1. Hall A. Rho GTPases and the actin cytoskeleton. Science $1998 ; 279: 509-14$.

2. Burridge K, Wennerberg K. Rho and Rac take center stage. Cell $2004 ; 116: 167-79$.

3. Vetter IR, Wittinghofer $A$. The guanine nucleotide-binding switch in three dimensions. Science $2001 ; 294: 1299-304$.

4. Valencia $A$, Chardin $P$, Wittinghofer $A$, Sander $C$. The ras protein family: evolutionary tree and role of conserved amino acids. Biochemistry $1991 ; 30: 4637-48$.

5. Williams CL. The polybasic region of Ras and Rho family small GTPases: a regulator of protein interactions and membrane association and a site of nuclear localization signal sequences. Cell Signal 2003; $15: 1071-80$.

6. Armstrong SA, Hannah VC, Goldstein JL, Brown MS. CAAX geranylgeranyl transferase transfers farnesyl as efficiently as geranylgeranyl to RhoB. J Biol Chem 1995 ; 270 : 7864-8.

7. Sahai $\varepsilon$, Marshall CJ. RHO-GTPases and cancer. Nat Rev Cancer $2002 ; 2: 133-42$.

8. Olson MF, Paterson HF, Marshall CJ. Signals from Ras and Rho GTPases interact to regulate expression of p21Wafl/Cipl. Nature $1998 ; 394: 295-9$.

9. Adnane J, Muro-Cacho C, Mathews L, Sebti SM, Munoz-Antonia T. Suppression of rho B expression in invasive carcinoma from head and neck cancer patients. Clin Cancer Res 2002 ; $8: 2225-32$.

10. Zalcman G, Closson V, Linares-Cruz G, et al. Regulation of Ras-related RhoB protein expression during the cell cycle. Oncogene $1995 ; 10: 1935-45$.

11. Knaus UG, Wang Y, Reilly AM, Warnock D, Jackson JH. Structural requirements for PAK activation by Rac GTPases. J Biol Chem 1998 ; 273 : 21512-8.

12. Haataja L, Groffen J, Heisterkamp N. Characterization of RAC3, a novel member of the Rho family. J Biol Chem $1997 ; 272: 20384-8$.

13. Roberts AW, Kim C, Zhen L, et al. Deficiency of the hematopoietic cell-specific Rho family GTPase Rac2 is characterized by abnormalities in neutrophil function and host defense. Immunity $1999 ; 10: 183-96$.

14. Katoh H, Negishi M. RhoG activates Racl by direct interaction with the Dock180-binding protein Elmo. Nature 2003; $424: 461-4$.

15. Nakaya M, Tanaka M, Okabe Y, Hanayama R, Nagata S. Opposite effects of rho family GTPases on engulfment of apoptotic cells by macrophages. J Biol Chem 2006; $281: 8836-42$.
16. Michaelson D, Silletti J, Murphy G, et al. Differential localization of Rho GTPases in live cells: regulation by hypervariable regions and RhoGDI binding. J Cell Biol 2001; 152 : 111-26.

17. Tanabe K, Tachibana T, Yamashita T, et al. The small GTP-binding protein TC10 promotes nerve elongation in neuronal cells, and its expression is induced during nerve regeneration in rats. J Neurosci $2000 ; 20: 4138-44$

18. Erschbamer MK, Hofstetter CP, Olson L. RhoA, RhoB, RhoC, Racl, Cdc42, and Tc10 mRNA levels in spinal cord, sensory ganglia, and corticospinal tract neurons and long-lasting specific changes following spinal cord injury. J Comp Neurol 2005 ; 484 : 224-33.

19. Chiang SH, Baumann CA, Kanzaki M, et al. Insulin-stimulated GLUT4 translocation requires the CAP-dependent activation of TC10. Nature $2001 ; 410: 944-8$.

20. De Toledo M, Senic-Matuglia F, Salamero J, et al. The GTP/GDP cycling of rho GTPase TCL is an essential regulator of the early endocytic pathway. Mol Biol Cell $2003 ; 14: 4846-56$.

21. Tao W, Pennica D, Xu L, et al. Wrch-1, a novel member of the Rho gene family that is regulated by Wnt-1. Genes Dev 2001; 15: 1796-807.

22. Chenette $\varepsilon$ J, Abo A, Der CJ. Critical and distinct roles of amino- and carboxyl-terminal sequences in regulation of the biological activity of the Chp atypical Rho GTPase. J Biol Chem 2005 ; 280 : 13784-92.

23. Shutes A, Berzat AC, Cox AD, Der CJ. Atypical mechanism of regulation of the Wrch-1 Rho family small GTPase. Curr Biol 2004 ; 14 : 2052-6.

24. Chardin P. Function and regulation of Rnd proteins. Nat Rev Mol Cell Biol $2006 ; 7: 54-62$.

25. Foster R, Hu Ke, Lu Y, et al. Identification of a novel human Rho protein with unusual properties: GTPase deficiency and in vivo farnesylation. Mol Cell Biol 1996; $16: 2689-99$.

26. Nobes CD, Lauritzen I, Mattei MG, et al. A new member of the Rho family, Rndl, promotes disassembly of actin filament structures and loss of cell adhesion. J Cell Biol 1998; 141 : 187-97.

27. Wennerberg K, Forget MA, Ellerbroek SM, et al. Rnd proteins function as RhoA antagonists by activating pl90 RhoGAP. Curr Biol 2003; 13: 1106-15.

28. Oinuma I, Ishikawa $Y$, Katoh H, Negishi M. The Semaphorin $4 D$ receptor Plexin-Bl is a GTPase activating protein for R-Ras. Science 2004 ; 305: 862-5.

29. Aspenstrom P, Fransson A, Saras J. Rho GTPases have diverse effects on the organization of the actin filament system. Biochem / 2004 ; $377: 327-37$.

30. Gasman S, Kalaidzidis Y, Zerial M. RhoD regulates endosome dynamics through diaphanous-related formin and Src tyrosine kinase. Nat Cell Biol $2003 ; 5: 195-204$.

31. Ellis $\mathrm{S}$, Mellor $H$. The novel Rho-family GTPase rif regulates coordinated actin-based membrane rearrangements. Curr Biol $2000 ; 10$ : 1387-90.

32. Rivero F, Dislich H, Glockner G, Noegel AA. The Dictyostelium discoideum family of Rho-related proteins. Nucleic Acids Res $2001 ; 29$ : 1068-79.

33. Siripurapu V, Meth J, Kobayashi N, Hamaguchi M. DBC2 significantly influences cell-cycle, apoptosis, cytoskeleton and membrane-trafficking pathways. J Mol Biol 2005 ; $346: 83-9$.

34. St-Pierre B, Jiang Z, Egan $S E$, Zacksenhaus $\varepsilon$. High expression during neurogenesis but not mammogenesis of a murine homologue of the deleted in breast cancer2/Rhobtb2 tumor suppressor. Gene Expr Patterns $2004 ; 5: 245-51$.

35. Cherry LK, Li X, Schwab P, et al. RhoH is required to maintain the integrin LFA-1 in a nonadhesive state on lymphocytes. Nat Immunol 2004 ; $5: 961-7$.

\section{TIRÉS À PART}

N. Lamarche-Vane 